\title{
The existence of solutions for elliptic systems with nonuniform growth
}

by

\author{
YONGQIANG Fu (Harbin)
}

\begin{abstract}
We study the Dirichlet problems for elliptic partial differential systems with nonuniform growth. By means of the Musielak-Orlicz space theory, we obtain the existence of weak solutions, which generalizes the result of Acerbi and Fusco [1].
\end{abstract}

1. Introduction. Let $\Omega \subset \mathbb{R}^{n}$ be a bounded Lipschitz domain. It is our purpose to study the following systems:

$$
\begin{aligned}
& \frac{\partial A_{\alpha}^{i}}{\partial x^{\alpha}}(x, u(x), D u(x))=B^{i}(x, u(x), D u(x)), \quad x \in \Omega, i=1, \ldots, N, \\
& u^{i}(x)=0, \quad x \in \partial \Omega, i=1, \ldots, N,
\end{aligned}
$$

where $u: \Omega \rightarrow \mathbb{R}^{N}$ is a vector-valued function. We use the summation convention throughout with $i, j$ running from 1 to $N$ and $\alpha, \beta$ running from 1 to $n$.

Because problems with nonuniform growth have important applications in mechanics, in recent years numerous papers have been devoted to the study of elliptic equations with nonuniform growth (see [2], [3], [7]-[10], [13], $[14],[16]$ and the references therein). The results of these papers show that problems with nonuniform growth conditions are much more complicated than those with standard growth conditions. These works motivate our study of the Dirichlet problem (1.1)-(1.2) in the setting of Musielak-Orlicz spaces.

In this paper, we suppose that the coefficients of (1.1) satisfy:

(H1) $\quad A_{\alpha}^{i}: \Omega \times \mathbb{R}^{N} \times M^{N \times n} \rightarrow \mathbb{R}, B^{i}: \Omega \times \mathbb{R}^{N} \times M^{N \times n} \rightarrow \mathbb{R}$ are Carathéodory functions, $i=1, \ldots, N, \alpha=1, \ldots, n$.

(H2) $\quad|A(x, s, \xi)| \leq C_{1}|\xi|^{p(x)-1}+C_{2}|s|^{p(x)-1}+G(x)$, where $G \in L^{p^{\prime}(\cdot)}(\Omega)$, $C_{1}, C_{2} \geq 0$ and $C_{2}$ small.

$$
\begin{aligned}
& |B(x, s, \xi)| \leq C_{1}^{\prime}|\xi|^{p(x)-1}+C_{2}^{\prime}|s|^{p(x)-1}+\bar{G}(x), \text { where } \bar{G} \in L^{p^{\prime}(\cdot)}(\Omega), \\
& C_{1}^{\prime}, C_{2}^{\prime} \geq 0 \text { and small. }
\end{aligned}
$$

2000 Mathematics Subject Classification: Primary 35B65.

This work is supported by Y. S. F. of Harbin and F. of HIT. 
(H4) $\quad A_{\alpha}^{i}(x, s, \xi) \xi_{\alpha}^{i} \geq \lambda_{0}|\xi|^{p(x)}-C|s|^{p(x)}+h(x)$, where $\lambda_{0}>0, C \geq 0$ small and $h \in L^{1}(\Omega)$.

(H5) For almost every $x_{0} \in \Omega, s_{0} \in \mathbb{R}^{N}$, the mapping $\xi \mapsto A\left(x_{0}, s_{0}, \xi\right)$ satisfies

$$
\int_{G} A_{\alpha}^{i}\left(x_{0}, s_{0}, \xi_{0}+D z(x)\right) z_{, \alpha}^{i}(x) d x \geq \nu \int_{G}|D z(x)|^{p(x)} d x
$$

for each $\xi_{0} \in M^{N \times n}, G \subset \mathbb{R}^{n}, z \in C_{0}^{1}\left(G, \mathbb{R}^{N}\right)$ where $\nu>0$ and $(D u(x))_{\alpha}^{i}=\partial u^{i}(x) / \partial x^{\alpha}=u_{, \alpha}^{i}(x)$.

Here $p: \Omega \rightarrow[1, \infty]$ is a measurable function and $p^{\prime}$ is its conjugate function (see Section 2).

For a simple case of (1.1), the Euler-Lagrange systems:

$\sum_{\alpha=1}^{n} \frac{\partial}{\partial x^{\alpha}} F_{u_{, \alpha}^{i}}^{i}(x, u(x), D u(x))-F_{u}^{i}(x, u(x), D u(x))=0, x \in \Omega, i=1, \ldots, N$,

which can be reduced to finding the stationary points of the functional

$$
\int_{\Omega} F^{i}(x, u(x), D u(x)) d x, \quad i=1, \ldots, N,
$$

it is immediate to obtain the existence of weak solutions in Sobolev spaces by applying Acerbi and Fusco [1]. From this point of view, the existence of weak solutions for (1.1) in a Musielak-Orlicz space (Theorem 3.1) is a generalization of their result.

\section{Preliminaries}

DEFinition 2.1. Let $M^{N \times n}$ be the set of real $N \times n$ matrices. A function $f: \mathbb{R}^{n} \times \mathbb{R}^{N} \times M^{N \times n} \rightarrow \mathbb{R}$ is called a Carathéodory function if it satisfies: for all $(s, \xi) \in \mathbb{R}^{N} \times M^{N \times n}, x \mapsto f(x, s, \xi)$ is measurable; for almost every $x \in \mathbb{R}^{n},(s, \xi) \mapsto f(x, s, \xi)$ is continuous.

Lemma 2.1 (see [6]). $f: \mathbb{R}^{n} \times \mathbb{R}^{N} \times M^{N \times n} \rightarrow \mathbb{R}$ is a Carathéodory function if and only if for each compact set $K \subset \mathbb{R}^{n}$ and every $\varepsilon>0$, there exists a compact set $K_{\varepsilon} \subset K$ satisfying meas $\left(K \backslash K_{\varepsilon}\right)<\varepsilon$ such that $f$ is continuous on $K_{\varepsilon} \times \mathbb{R}^{N} \times M^{N \times n}$.

Lemma 2.2 (see [5]). Let $G \subset \mathbb{R}^{n}$ be measurable and meas $(G)<\infty$. Suppose that $\left\{M_{k}\right\}$ is a sequence of subsets of $G$ such that for some $\varepsilon>0$, $\operatorname{meas}\left(M_{k}\right) \geq \varepsilon \quad$ for each $k \in \mathbb{N}$.

Then there exists a subsequence $\left\{M_{k_{h}}\right\}$ such that $\bigcap_{h \in \mathbb{N}} M_{k_{h}} \neq \emptyset$.

LEMMA 2.3 (see [1]). Let $\left\{f_{k}\right\}$ be a sequence of bounded functions in $L^{1}\left(\mathbb{R}^{n}\right)$. For each $\varepsilon>0$ there exists $\left(A_{\varepsilon}, \delta, S\right)$ (where $A_{\varepsilon}$ is measurable and 
meas $\left(A_{\varepsilon}\right)<\varepsilon, \delta>0, S$ is an infinite subset of $\left.\mathbb{N}\right)$ such that for each $k \in S$,

$$
\int_{B}\left|f_{k}(x)\right| d x<\varepsilon
$$

where $B$ and $A_{\varepsilon}$ are disjoint and meas $(B)<\delta$.

Definition 2.2. For $u \in C_{0}^{1}\left(\mathbb{R}^{n}\right)$, define

$$
\left(M^{*} u\right)(x)=(M u)(x)+\sum_{\alpha=1}^{n}\left(M D_{\alpha} u\right)(x)
$$

where

$$
(M u)(x)=\sup _{r>0} \frac{1}{\operatorname{meas}\left(B_{r}(x)\right)} \int_{B_{r}(x)} f(x) d x,
$$

$B_{r}(x)=\left\{y \in \mathbb{R}^{n}:|y-x|<r\right\}$ and $D_{\alpha} u=\partial u / \partial x^{\alpha}$.

Lemma 2.4 (see [12]). If $u \in C_{0}^{\infty}\left(\mathbb{R}^{n}\right)$, then $M^{*} u \in C^{0}\left(\mathbb{R}^{n}\right)$ and for all $x \in \mathbb{R}^{n}$,

$$
|u(x)|+\sum_{\alpha=1}^{n}\left|D_{\alpha} u(x)\right| \leq\left(M^{*} u\right)(x) .
$$

Furthermore, if $p>1$, then

$$
\left\|M^{*} u\right\|_{L^{p}\left(\mathbb{R}^{n}\right)} \leq C(n, p)\|u\|_{W_{0}^{1, p}\left(\mathbb{R}^{n}\right)}
$$

and if $p=1$, then

$$
\operatorname{meas}\left(\left\{x \in \mathbb{R}^{n}:\left(M^{*} u\right)(x) \leq \lambda\right\}\right) \leq \frac{C(n)}{\lambda}\|u\|_{W^{1,1}\left(\mathbb{R}^{n}\right)}
$$

for all $\lambda>0$.

Lemma 2.5 (see [12]). Let $u \in C_{0}^{\infty}\left(\mathbb{R}^{n}\right)$. Define

$$
U(x, y)=\frac{\left|u(y)-u(x)-\sum_{\alpha=1}^{n} D_{\alpha} u(x)\left(y^{\alpha}-x^{\alpha}\right)\right|}{|y-x|} .
$$

For all $x \in \mathbb{R}^{n}, r>0$, we have

$$
\int_{B_{r}(x)} U(x, y) d y \leq 2 \operatorname{meas}\left(B_{r}(x)\right)\left(M^{*} u\right)(x) .
$$

Lemma 2.6 (see [1]). Let $u \in C_{0}^{\infty}\left(\mathbb{R}^{n}\right)$ and $\lambda>0$. Set

$$
H^{\lambda}=\left\{x \in \mathbb{R}^{n}:\left(M^{*} u\right)(x)<\lambda\right\} .
$$

Then for all $x, y \in H^{\lambda}$, we have

$$
|u(y)-u(x)| \leq C(n) \lambda|y-x| .
$$

Lemma 2.7 (see [15]). Let $X$ be a metric space, $E$ a subspace of $X$, and $k$ a positive number. Then any $k$-Lipschitz mapping from $E$ into $\mathbb{R}$ can be extended to a $k$-Lipschitz mapping from $X$ into $\mathbb{R}$. 
Let $\mathcal{P}(\Omega)$ be the family of all Lebesgue measurable functions $p(\cdot)$ : $\Omega \rightarrow[1, \infty]$. For $p(\cdot) \in \mathcal{P}(\Omega)$, we put $\Omega_{1}^{p(\cdot)}=\{x \in \Omega: p(x)=1\}, \Omega_{\infty}^{p(\cdot)}=$ $\{x \in \Omega: p(x)=\infty\}, \Omega_{0}^{p(\cdot)}=\Omega \backslash\left(\Omega_{1}^{p(\cdot)} \cup \Omega_{\infty}^{p(\cdot)}\right), p_{*}=\operatorname{essinf}_{x \in \Omega_{0}^{p(\cdot)}} p(x)$ and $p^{*}=\operatorname{essup}_{\Omega_{0}^{p(\cdot)}} p(x)$ if meas $\left(\Omega_{0}^{p(\cdot)}\right)>0, p_{*}=p^{*}=1$ if $\operatorname{meas}\left(\Omega_{0}^{p(\cdot)}\right)=0$. We use the convention $1 / \infty=0$.

Let $p(\cdot) \in \mathcal{P}(\Omega)$. On the set of all functions on $\Omega$, we define $\varrho_{p(\cdot)}$ and $\|\cdot\|_{L^{p(\cdot)}(\Omega)}$ by

$$
\begin{gathered}
\varrho_{p(\cdot)}(f)=\int_{\Omega \backslash \Omega_{\infty}^{p(\cdot)}}|f(x)|^{p(x)} d x+\underset{x \in \Omega_{\infty}^{p(\cdot)}}{\operatorname{esssup}}|f(x)|, \\
\|f\|_{L^{p(\cdot)}(\Omega)}=\inf \left\{\lambda>0: \varrho_{p(\cdot)}(f / \lambda) \leq 1\right\} .
\end{gathered}
$$

The space $L^{p(\cdot)}(\Omega)$ is the class of all functions $f$ such that $\varrho_{p(\cdot)}(\lambda f)<\infty$ for some $\lambda=\lambda(f)>0$. Thus $L^{p(\cdot)}(\Omega)$ is a Musielak-Orlicz space.

Given $p(\cdot) \in \mathcal{P}(\Omega)$, we define the conjugate function $p^{\prime}(\cdot) \in \mathcal{P}(\Omega)$ by

$$
p^{\prime}(x)= \begin{cases}\infty & \text { if } x \in \Omega_{1}^{p(\cdot)}, \\ 1 & \text { if } x \in \Omega_{\infty}^{p(\cdot)}, \\ \frac{p(x)}{p(x)-1} & \text { if } x \in \Omega_{0}^{p(\cdot)}\end{cases}
$$

Lemma 2.8. Let $p(\cdot) \in \mathcal{P}(\Omega)$. Then

$$
\int_{\Omega}|f(x) g(x)| d x \leq C(p(\cdot))\|f\|_{L^{p(\cdot)}(\Omega)}\|g\|_{L^{p^{\prime}(\cdot)}(\Omega)}
$$

for every $f \in L^{p(\cdot)}(\Omega)$ and $g \in L^{p^{\prime}(\cdot)}(\Omega)$.

We shall say that $\left\{f_{n}\right\} \subseteq L^{p(\cdot)}(\Omega)$ converges modularly to a function $f \in L^{p(\cdot)}(\Omega)$ if $\lim _{n \rightarrow \infty} \varrho_{p(\cdot)}\left(f-f_{n}\right)=0$.

Lemma 2.9. (1) The topology of $L^{p(\cdot)}(\Omega)$ given by the norm coincides with the topology of modular convergence if and only if $p^{*}<\infty$.

(2) $L^{p(\cdot)}(\Omega)$ is complete.

(3) The dual space to $L^{p(\cdot)}(\Omega)$ is $L^{p^{\prime}(\cdot)}(\Omega)$ if and only if $p(\cdot) \in L^{\infty}(\Omega)$.

(4) The space $L^{p(\cdot)}(\Omega)$ is reflexive if and only if $1<p_{*} \leq p^{*}<\infty$.

Lemma 2.10. Let $p(\cdot) \in \mathcal{P}(\Omega) \cap L^{\infty}(\Omega)$.

(1) $C_{0}^{\infty}(\Omega)$ is dense in $L^{p(\cdot)}(\Omega)$,

(2) $L^{p(\cdot)}(\Omega)$ is separable. 
Given a multiindex $\alpha=\left(\alpha_{1}, \ldots, \alpha_{N}\right) \in \mathbb{N}^{N}$, we set $|\alpha|=\alpha_{1}+\ldots+\alpha_{N}$ and $D^{\alpha}=D_{1}^{\alpha_{1}} \ldots D_{N}^{\alpha_{N}}$, where $D_{i}=\partial / \partial x_{i}$ is the generalized derivative operator.

The space $W^{k, p(\cdot)}(\Omega)$ is the class of all functions $f$ on $\Omega$ such that $D^{\alpha} f \in L^{p(\cdot)}(\Omega)$ for every multiindex $\alpha$ with $|\alpha| \leq k$, endowed with the norm

$$
\|f\|_{W^{k, p(\cdot)}(\Omega)}=\sum_{|\alpha| \leq k}\left\|D^{\alpha} f\right\|_{W^{k, p(\cdot)}(\Omega)} .
$$

We denote by $W_{0}^{k, p(\cdot)}(\Omega)$ the subspace of $W^{k, p(\cdot)}(\Omega)$ which is the closure of $C_{0}^{\infty}(\Omega)$ with respect to the norm of $W^{k, p(\cdot)}(\Omega)$.

LEMma 2.11. $W^{k, p(\cdot)}(\Omega)$ and $W_{0}^{k, p(\cdot)}(\Omega)$ are Banach spaces, which are separable if $p(\cdot) \in L^{\infty}(\Omega)$ and reflexive if $p(\cdot)$ satisfies

$$
1<p_{*} \leq p^{*}<\infty .
$$

We shall say that a function $p(\cdot) \in \mathcal{P}(\Omega)$ is $*$-continuous on $\Omega$ if

$$
\lim _{y \rightarrow x, y \in \Omega} p(y)=p(x) \quad \text { for every } x \in \Omega
$$

(i.e. even if $p(x)=\infty$ ).

Throughout this paper, we suppose that $p(\cdot)$ is $*$-continuous on $\bar{\Omega}$ and $p(\cdot) \in L^{\infty}(\Omega)$.

Lemma 2.12. (1) Let $p(\cdot), q(\cdot) \in \mathcal{P}(\Omega)$. If $q(x) \leq p(x)$ for a.e. $x \in \Omega$, then the embedding $W^{k, p(\cdot)}(\Omega) \subseteq W^{k, q(\cdot)}(\Omega)$ is continuous.

(2) Let $p(\cdot) \in \mathcal{P}(\Omega)$. If $p(\cdot)$ is $*$-continuous on $\bar{\Omega}$, then the embedding $W_{0}^{k, p(\cdot)}(\Omega) \subseteq L^{p(\cdot)}(\Omega)$ is compact.

Lemma 2.13. Let $p(\cdot) \in \mathcal{P}(\Omega) \cap L^{\infty}(\Omega)$. Then for every $G$ in the dual space $\left(W_{0}^{k, p(\cdot)}(\Omega)\right)^{*}$, there exists a unique system $\left\{g_{\alpha} \in L^{p^{\prime}(\cdot)}(\Omega):|\alpha| \leq k\right\}$ of functions such that

$$
\langle G, f\rangle=\sum_{|\alpha| \leq k} \int_{\Omega} D^{\alpha} f(x) g_{\alpha}(x) d x, \quad f \in W_{0}^{k, p(\cdot)}(\Omega) .
$$

In view of Lemma 2.13, we denote $\left(W_{0}^{k, p(\cdot)}(\Omega)\right)^{*}$ by $W^{-k, p^{\prime}(\cdot)}(\Omega)$ and endow it with the norm

$$
\|v\|_{W^{-k, p^{\prime}(\cdot)}(\Omega)}=\sup _{u \in W_{0}^{k, p(\cdot)}(\Omega)}|\langle u, v\rangle| .
$$

We refer to O. Kováčik and J. Rákosnik [11] for the notions and lemmas mentioned above. 
LEMMA 2.14. If $p(\cdot) \in L^{\infty}(\Omega)$ and $u \in W_{0}^{1, p(\cdot)}(\Omega)$, then

$$
\int_{\Omega}|u|^{p(x)} d x \leq C \int_{\Omega}|D u|^{p(x)} d x
$$

where $C$ is a constant depending on $\Omega$.

Proof. Set $R=\operatorname{diam} \Omega$. By translation, we may assume that $0<x^{n}<R$ in $\Omega$. Then we can extend $u$ to be zero outside $\Omega$, so

$$
u(x)=\int_{0}^{x^{n}} D_{n} u\left(x^{\prime}, t\right) d t \quad \text { a.e. } x=\left(x^{\prime}, x^{n}\right) \in \Omega .
$$

Integrating with respect to $x^{n}$, we have

$$
\begin{aligned}
& \int_{0}^{R}\left(\frac{\left|u\left(x^{\prime}, x^{n}\right)\right|}{R}\right)^{p(x)} d x^{n} \\
& \leq \int_{0}^{R}\left(\int_{0}^{x^{n}} \frac{\left|D_{n} u\left(x^{\prime}, t\right)\right|}{R} d t\right)^{p(x)} d x^{n} \\
& \leq \int_{0}^{R} \int_{0}^{x^{n}}\left(\frac{x^{n}\left|D_{n} u\left(x^{\prime}, t\right)\right|}{R}\right)^{p(x)} d t\left|x^{n}\right|^{p(x)-1} d x^{n} \\
& \leq C \int_{0}^{R} \int_{0}^{R}\left|D_{n} u\left(x^{\prime}, t\right)\right|^{p(x)} d t d x^{n}=C \int_{0}^{R} \int_{t}^{R}\left|D_{n} u\left(x^{\prime}, t\right)\right|^{p(x)} d x^{n} d t \\
& \quad=C \int_{0}^{R}(R-t)\left|D_{n} u\left(x^{\prime}, t\right)\right|^{p(x)} d t \leq C \int_{0}^{R}\left|D_{n} u\left(x^{\prime}, t\right)\right|^{p(x)} d t \\
& =C \int_{0}^{R}\left|D_{n} u(x)\right|^{p(x)} d x^{n} .
\end{aligned}
$$

Finally we integrate with respect to $x^{\prime}$ over $\mathbb{R}^{n-1}$ and the conclusion follows.

Lemma 2.15. If $p(\cdot) \in L^{\infty}(\Omega)$, then

$$
\lim _{\operatorname{meas}(E) \rightarrow 0}\left\|u \chi_{E}\right\|_{L^{p(\cdot)}(\Omega)}=0
$$

for all $u \in L^{p(\cdot)}(\Omega)$.

Proof. By Lemma 2.10, for each $\varepsilon>0$ and each $u \in L^{p(\cdot)}(\Omega)$ there exists $w \in C_{0}^{\infty}(\Omega)$ such that $\|u-w\|_{L^{p(\cdot)}(\Omega)}<\varepsilon$. Suppose that $|w(x)| \leq C$ for all $x \in \Omega$. Let meas $(E)<1$. Then $\left\|\chi_{E}\right\|_{L^{p(\cdot)}(\Omega)} \leq(\operatorname{meas}(E))^{1 / p^{*}} \rightarrow 0$ as meas $(E) \rightarrow 0$. So there exists $\delta>0$ such that if meas $(E)<\delta$, then 
$\left\|\chi_{E}\right\|_{L^{p(\cdot)}(\Omega)}<\varepsilon /(2 C)$. Now we get

$$
\begin{aligned}
\left\|u \chi_{E}\right\|_{L^{p(\cdot)}(\Omega)} & \leq\left\|(u-w) \chi_{E}\right\|_{L^{p(\cdot)}(\Omega)}+\left\|w \chi_{E}\right\|_{L^{p(\cdot)}(\Omega)} \\
& \leq\|u-w\|_{L^{p(\cdot)}(\Omega)}+C\left\|\chi_{E}\right\|_{L^{p(\cdot)}(\Omega)}<\varepsilon,
\end{aligned}
$$

that is to say, $\lim _{\text {meas }(E) \rightarrow 0}\left\|u \chi_{E}\right\|_{L^{p(\cdot)}(\Omega)}=0$.

Lemma 2.16. Suppose that $p(\cdot) \in L^{\infty}(\Omega)$. Let $\left\{u_{k}\right\}_{k=1}^{\infty}$ be bounded in $L^{p(\cdot)}(\Omega)$. If $u_{k} \rightarrow u$ a.e. on $\Omega$, then $u_{k} \rightarrow u$ weakly in $L^{p(\cdot)}(\Omega)$.

Proof. Suppose that $\left\|u_{k}\right\|_{L^{p(\cdot)}(\Omega)} \leq C$ for each integer $k$. By Fatou's Lemma,

$$
\int_{\Omega}\left(\frac{u}{C}\right)^{p(x)} d x=\int_{\Omega} \lim _{k \rightarrow \infty}\left(\frac{u_{k}}{C}\right)^{p(x)} d x \leq \liminf _{k \rightarrow \infty} \int_{\Omega}\left(\frac{u_{k}}{C}\right)^{p(x)} d x \leq 1,
$$

hence $\|u\|_{L^{p(\cdot)}(\Omega)} \leq C$. Let $\varepsilon>0$ and $g \in L^{p^{\prime}(\cdot)}(\Omega)$. By Lemma 2.15, $\lim _{\text {meas }(E) \rightarrow 0}\left\|g \chi_{E}\right\|_{L^{p^{\prime}(\cdot)(\Omega)}}=0$ and so there exists $\delta>0$ such that for all $E$ satisfying meas $(E)<\delta$, we have

$$
\left\|g \chi_{E}\right\|_{L^{p^{\prime}(\cdot)}(\Omega)}<\frac{\varepsilon}{4 C} .
$$

By Egorov's Theorem, there exists a set $B$ such that $u_{k} \rightarrow u$ uniformly on $B$ and meas $(\Omega \backslash B)<\delta$. Finally choose $K$ such that $k>K$ implies

$$
\max _{x \in B}\left|u-u_{k}\right| \cdot\|g\|_{L^{p^{\prime}(\cdot)}(\Omega)}\left\|\chi_{\Omega}\right\|_{L^{p(\cdot)}(\Omega)}<\frac{\varepsilon}{2}
$$

for all $x \in B$. Thus taking $E=\Omega \backslash B$, we have

$$
\begin{aligned}
& \left|\int_{\Omega} u g d x-\int_{\Omega} u_{k} g d x\right| \leq \int_{B}\left|u_{k}-u\right| \cdot|g| d x+\int_{\Omega \backslash B}\left|u_{k}-u\right| \cdot|g| d x \\
& \leq\|g\|_{L^{p^{\prime}(\cdot)}(\Omega)}\left\|\chi_{\Omega}\right\|_{L^{p(\cdot)}(\Omega)} \max _{x \in B}\left|u_{k}-u\right|+\left\|u_{k}-u\right\|_{L^{p(\cdot)}(\Omega)}\left\|g \chi_{\Omega \backslash B}\right\|_{L^{\left.p^{(} \cdot\right)}(\Omega)}<\varepsilon
\end{aligned}
$$
for all $k>K$, that is to say, $u_{k} \rightarrow u$ weakly in $L^{p(\cdot)}(\Omega)$.

\section{Main theorem}

Theorem 3.1. Under the conditions (H1)-(H5), the Dirichlet problem (1.1)-(1.2) has at least one weak solution in $W_{0}^{1, p(\cdot)}\left(\Omega, \mathbb{R}^{N}\right)$, that is to say, there exists at least one $u \in W_{0}^{1, p(\cdot)}\left(\Omega, \mathbb{R}^{N}\right)$ satisfying

$$
\int_{\Omega}\left[A_{\alpha}^{i}(x, u, D u) z_{, \alpha}^{i}(x)+B^{i}(x, u, D u) z^{i}(x)\right] d x=0
$$

for all $z \in W_{0}^{1, p(\cdot)}\left(\Omega, \mathbb{R}^{N}\right)$. 
Proof. Set $V=W_{0}^{1, p(\cdot)}\left(\Omega, \mathbb{R}^{N}\right)$. For $u \in V$, define $T: V \rightarrow V^{*}$ in the following way: for each $w \in V$,

$$
(T u, w)=\int_{\Omega}\left[A_{\alpha}^{i}(x, u, D u) w_{, \alpha}^{i}(x)+B^{i}(x, u, D u) w^{i}(x)\right] d x=0 .
$$

Now we only need to show that there exists $u \in V$ such that $(T u, w)=0$ for all $w \in V$. We will prove this in several steps.

1) $T$ is strong-weakly continuous. Suppose that $u_{k} \rightarrow u$ strongly in $W_{0}^{1, p(\cdot)}\left(\Omega, \mathbb{R}^{N}\right)$. Then $\|u\|_{V} \leq C$ for some constant $C$ independent of $k$. By (H2)-(H3), $A_{\alpha}^{i}\left(x, u_{k}, D u_{k}\right)$ and $B^{i}\left(x, u_{k}, D u_{k}\right)$ are bounded in $L^{p^{\prime}(\cdot)}(\Omega)$. Then by (H1) and Lemma 2.16, we know

$$
\lim _{k \rightarrow \infty}\left(T u_{k}, w\right)=\left(T\left(\lim _{k \rightarrow \infty} u_{k}\right), w\right)=(T u, w) .
$$

That is to say, $T$ is strong-weakly continuous.

2) $T$ is coercive, i.e.

$$
\lim _{\|u\|_{V} \rightarrow \infty} \frac{(T u, u)}{\|u\|_{V}}=+\infty .
$$

By (H1)-(H2) and Lemma 2.14,

$$
\begin{aligned}
(T u, u) \geq & \int_{\Omega}\left[\lambda_{0}|D u|^{p(x)}-C|u|^{p(x)}+h(x)-C_{1}^{\prime}|D u|^{p(x)-1}|u|\right. \\
& \left.-C_{2}^{\prime}|u|^{p(x)}-\bar{G}(x)|u|\right] d x \\
\geq & \int_{\Omega}\left[\lambda_{0}|D u|^{p(x)}-C|u|^{p(x)}+h(x)-C_{1}^{\prime}|D u|^{p(x)}-C_{1}^{\prime}|u|^{p(x)}\right. \\
& \left.-C_{2}^{\prime}|u|^{p(x)}-\mu|u|^{p(x)}-C(\mu)(\bar{G}(x))^{p^{\prime}(x)}\right] d x \\
\geq & \int_{\Omega}\left[\left(\lambda_{0}-C_{1}^{\prime}-C^{*}\left(C+C_{1}^{\prime}+C_{2}^{\prime}+\mu\right)\right)|D u|^{p(x)}\right. \\
& \left.+h(x)-C(\mu)(\bar{G}(x))^{p^{\prime}(x)}\right] d x
\end{aligned}
$$

where $C^{*}$ is the constant in Lemma 2.14 .

When $C, C_{1}^{\prime}, C_{2}^{\prime}, \mu$ are small, we can get

$$
\lambda_{0}-C_{1}^{\prime}-C^{*}\left(C+C_{1}^{\prime}+C_{2}^{\prime}+\mu\right)>0 .
$$

By Lemma 2.12, we have

$$
\left(1+C_{1}^{*}\right)\||D u|\|_{L^{p(\cdot)}(\Omega)} \geq\|u\|_{L^{p(\cdot)}\left(\Omega, \mathbb{R}^{N}\right)}+\||D u|\|_{L^{p(\cdot)}(\Omega)} \geq\|u\|_{V}
$$

where $C_{1}^{*}$ is the imbedding constant. In view of (3.5), it is easy to see that $\||D u|\|_{L^{p(\cdot)}(\Omega)} \rightarrow \infty$ as $\|u\|_{V} \rightarrow \infty$. Taking $\varepsilon$ sufficiently small, for example

$$
\varepsilon=\frac{1}{2}\left(\||D u|\|_{L^{p(\cdot)}(\Omega)}-e^{\left.\frac{2}{p *+1} \ln \||D u|\|_{L^{p(\cdot)}(\Omega)}\right),}\right.
$$


we have

$$
\begin{aligned}
\frac{\int_{\Omega}|D u|^{p(x)} d x}{\|u\|_{V}} & =\int_{\Omega}\left(\frac{|D u|}{\||D u|\|_{L^{p(\cdot)}(\Omega)}-\varepsilon}\left(\||D u|\|_{L^{p(\cdot)}(\Omega)}-\varepsilon\right)\right)^{p(x)} \frac{1}{\|u\|_{V}} d x \\
& \geq \int_{\Omega}\left(\frac{|D u|}{\||D u|\|_{L^{p(\cdot)}(\Omega)}-\varepsilon}\right)^{p(x)} d x \frac{\left(\||D u|\|_{L^{p(\cdot)}(\Omega)}-\varepsilon\right)^{p_{*}}}{\|u\|_{V}} \\
& \geq \frac{\left(\||D u|\|_{L^{p(\cdot)}(\Omega)}-\varepsilon\right)^{\left(p_{*}+1\right) / 2}}{\left(1+C_{1}^{*}\right)\|\mid D u\|_{L^{p(\cdot)}(\Omega)}}\left(\|\mid D u\|_{L^{p(\cdot)}(\Omega)}-\varepsilon\right)^{\left(p_{*}-1\right) / 2} \\
& \geq \frac{1}{1+C_{1}^{*}}\left(\||D u|\|_{L^{p(\cdot)}(\Omega)}-\varepsilon\right)^{\left(p_{*}-1\right) / 2} \rightarrow \infty
\end{aligned}
$$

as $\|u\|_{V} \rightarrow \infty$. As $\int_{\Omega}\left[h(x)-C(\mu)(\bar{G}(x))^{p^{\prime}(x)}\right] d x$ is bounded, we conclude that (3.4) holds.

3) Now we construct an approximating sequence. By Lemma 2.10, we can choose a basis $\left\{w_{k}\right\}$ of $V$ such that the union of subspaces finitely generated from $\left\{w_{k}\right\}$ is dense in $V$. Let $B_{s}$ be the subspace of $V$ generated by $w_{1}, \ldots, w_{s}$. By the coerciveness of $T$ and Morrey [15], there exists $u_{s} \in B_{s}$ such that

$$
\left(T u_{s}, w\right)=0
$$

for all $w \in B_{s}$. By the coerciveness of $T$ again, we know that $\left\|u_{s}\right\|_{V} \leq C$ where $C$ is independent of $s$. As $V$ is reflexive, we can extract a subsequence $\left\{u_{k}\right\}$ such that

$$
u_{k} \rightarrow u_{0} \text { weakly in } V, \quad T u_{k} \rightarrow \xi \text { weakly in } V^{*}, \quad(\xi, w)=0
$$

where $w$ is in a dense subset of $V$. For fixed $\xi$, by the continuity of $(\xi, \cdot)$, we get $(\xi, w)=0$ for all $w \in V$. Considering $\left(T u_{k}, u_{k}-u_{0}\right)$, we have

$$
\left(T u_{k}, u_{k}-u_{0}\right)=\left(T u_{k}, u_{k}\right)-\left(T u_{k}, u_{0}\right)=-\left(T u_{k}, u_{0}\right) \rightarrow 0
$$

as $k \rightarrow \infty$. Set $z_{k}=u_{k}-u_{0}$. Then

$$
z_{k} \rightarrow 0 \quad \text { weakly in } V \text { as } k \rightarrow \infty \text {. }
$$

Consider $\left(T u_{k}, u_{k}-u_{0}\right)$ once more:

$\left(T u_{k}, u_{k}-u_{0}\right)$

$$
=\int_{\Omega}\left[A_{\alpha}^{i}\left(x, u_{0}+z_{k}, D u_{0}+D z_{k}\right) z_{k, \alpha}^{i}+B^{i}\left(x, u_{0}+z_{k}, D u_{0}+D z_{k}\right) z_{k}^{i}\right] d x \rightarrow 0
$$

as $k \rightarrow \infty$. By applying Lemma 2.12, we get

$$
z_{k} \rightarrow 0 \quad \text { strongly in } L^{p(\cdot)}\left(\Omega, \mathbb{R}^{N}\right) .
$$

In view of (H3) and (3.6), it is immediate that

$$
\int_{\Omega} B^{i}\left(x, u_{0}+z_{k}, D u_{0}+D z_{k}\right) z_{k}^{i} d x \rightarrow 0
$$


as $k \rightarrow \infty$, that is to say,

$$
\int_{\Omega} A_{\alpha}^{i}\left(x, u_{0}+z_{k}, D u_{0}+D z_{k}\right) z_{k, \alpha}^{i} d x \rightarrow 0
$$

as $k \rightarrow \infty$.

Now if we can prove that there exists a subsequence of $\left\{z_{k}\right\}$ which is strongly convergent in $V$, then from the strong-weak continuity of $T$, we get $T u_{k} \rightarrow T u_{0}=\xi$ weakly in $V$ as $k \rightarrow \infty$ and $u_{0}$ will be a weak solution of (1.1) $-(1.2)$.

4) We will find a subsequence of $\left\{z_{k}\right\}$ which is strongly convergent in $V$. For each measurable set $S \subset \Omega$, define

$$
F(v, S)=\int_{S} A_{\alpha}^{i}\left(x, u_{0}+v, D u_{0}+D v\right) v_{, \alpha}^{i} d x
$$

where $v \in W_{0}^{1, p(\cdot)}\left(\Omega, \mathbb{R}^{N}\right)$. Similarly to the remark in step 1 , we can show $F(v, S)$ is strongly continuous in $W_{0}^{1, p(\cdot)}\left(\Omega, \mathbb{R}^{N}\right)$. Since $C_{0}^{\infty}\left(\Omega, \mathbb{R}^{N}\right)$ is dense in $W_{0}^{1, p(\cdot)}\left(\Omega, \mathbb{R}^{N}\right)$, there exists $\left\{f_{k}\right\} \subset C_{0}^{\infty}\left(\Omega, \mathbb{R}^{N}\right)$ such that

$$
\left\|f_{k}-z_{k}\right\|_{V}<1 / k, \quad\left|F\left(f_{k}, \Omega\right)-F\left(z_{k}, \Omega\right)\right|<1 / k .
$$

So we can suppose $\left\{z_{k}\right\}$ is in $C_{0}^{\infty}\left(\Omega, \mathbb{R}^{N}\right)$ and bounded in $W_{0}^{1, p(\cdot)}\left(\Omega, \mathbb{R}^{N}\right)$.

Next we define

$$
z_{k}(x)=0 \quad \text { when } x \in \mathbb{R}^{n} \backslash \Omega \text {. }
$$

In this way, we extend the domain of $z_{k}$ to $\mathbb{R}^{n}$ and $\left\{z_{k}\right\} \subset W_{0}^{1, p(x)}\left(\mathbb{R}^{n}, \mathbb{R}^{N}\right)$ and $\left\{z_{k}\right\}$ is bounded and $\operatorname{supp} z_{k} \subset \Omega$.

Let $\eta: \mathbb{R}^{+} \rightarrow \mathbb{R}^{+}$be a continuous increasing function satisfying $\eta(0)=0$ and for each measurable set $B \subset \Omega$,

$$
\begin{array}{r}
\sup _{k} \int_{B}\left[(g(x))^{p^{\prime}(x)}+h(x)+1+C\left(\left|u_{0}\right|^{p(x)}+\left|D u_{0}\right|^{p(x)}+\right.\right. \\
\left.\left.\left|z_{k}\right|^{p(x)}\right)\right] d x \\
\leq \eta(\operatorname{meas}(B))
\end{array}
$$

where $C=C_{1}+C_{2}$ and $C_{1}, C_{2}$ are the two constants in (H2).

Let $\left\{\varepsilon_{j}\right\}$ be a positive decreasing sequence with $\varepsilon_{j} \rightarrow 0$ as $j \rightarrow \infty$. For $\varepsilon_{1}$, applying Lemma 2.3 to each of the $N$ sequences $\left\{\left(M^{*} z_{k}^{i}\right)^{p(x)}\right\}, 1 \leq i \leq N$, we get a subsequence $\left\{z_{k_{1}}\right\}$, a set $A_{\varepsilon_{1}} \subset \Omega$ satisfying meas $\left(A_{\varepsilon_{1}}\right)<\varepsilon_{1}$, and a real number $\delta_{1}>0$ such that

$$
\int_{B}\left(M^{*} z_{k_{1}}^{i}\right)^{p(x)} d x<\varepsilon_{1}
$$

for all $k_{1}, 1 \leq i \leq N$ and $B \subset \Omega \backslash A_{\varepsilon_{1}}$ satisfying meas $(B)<\delta_{1}$. By Lemma 2.4 , we can choose $\lambda>1$ so large that for all $i$ and $k_{1}$,

$$
\operatorname{meas}\left(\left\{x \in \mathbb{R}^{n}:\left(M^{*} z_{k_{1}}^{i}\right)(x) \geq \lambda\right\}\right) \leq \min \left\{\varepsilon_{1}, \delta_{1}\right\} \text {. }
$$


For all $i$ and $k_{1}$, define

$$
H_{i, k_{1}}^{\lambda}=\left\{x \in \mathbb{R}^{n}:\left(M^{*} z_{k_{1}}^{i}\right)(x)<\lambda\right\}, \quad H_{k_{1}}^{\lambda}=\bigcap_{i=1}^{N} H_{i, k_{1}}^{\lambda} .
$$

By Lemma 2.6, we have

$$
\frac{\left|z_{k_{1}}^{i}(y)-z_{k_{1}}^{i}(x)\right|}{|y-x|} \leq C(n) \lambda
$$

for all $x, y \in H_{k_{1}}^{\lambda}$ and $1 \leq i \leq N$. From Lemma 2.7, there exists a Lipschitz function $g_{k_{1}}^{i}$ which extends $z_{k_{1}}^{i}$ outside $H_{k_{1}}^{\lambda}$ and the Lipschitz constant of $g_{k_{1}}^{i}$ is no more than $C(n) \lambda$. As $H_{k_{1}}^{\lambda}$ is an open set, we have $g_{k_{1}}^{i}(x)=z_{k_{1}}^{i}(x)$ and $D g_{k_{1}}^{i}(x)=D z_{k_{1}}^{i}(x)$ for all $x \in H_{k_{1}}^{\lambda}$, and

$$
\left\|\left|D g_{k_{1}}^{i}\right|\right\|_{L^{\infty}\left(\mathbb{R}^{n}\right)} \leq C(n) \lambda .
$$

In view of Lemma 2.4, we can further suppose that

$$
\left\|g_{k_{1}}^{i}\right\|_{L^{\infty}\left(\mathbb{R}^{n}\right)} \leq\left\|z_{k_{1}}^{i}\right\|_{L^{\infty}\left(H_{k_{1}}^{\lambda}\right)} \leq \lambda, \quad\left\|g_{k_{1}}\right\|_{W^{1, p(\cdot)\left(\Omega, \mathbb{R}^{N}\right)}} \leq C .
$$

By the boundedness of $\left\|g_{k_{1}}\right\|_{W^{1, \infty}\left(\Omega, \mathbb{R}^{N}\right)}$, there exists a subsequence of $\left\{g_{k_{1}}^{i}\right\}$ (still denoted by $\left\{g_{k_{1}}^{i}\right\}$ ) such that

$$
g_{k_{1}}^{i} \rightarrow v^{i} \quad * \text {-weakly in } W^{1, \infty}(\Omega) \text { as } k_{1} \rightarrow \infty
$$

for $1 \leq i \leq N$. Set $\left(g_{k_{1}}^{1}, \ldots, g_{k_{1}}^{N}\right)=g_{k_{1}}$ and $\left(v^{1}, \ldots, v^{N}\right)=v$. We have

$$
\begin{aligned}
F\left(z_{k_{1}}, \Omega\right)= & F\left(g_{k_{1}},\left(\Omega \backslash A_{\varepsilon_{1}}\right) \cap H_{k_{1}}^{\lambda}\right)+F\left(z_{k_{1}}, A_{\varepsilon_{1}} \cup\left(\Omega \backslash H_{k_{1}}^{\lambda}\right)\right) \\
= & F\left(g_{k_{1}}, \Omega \backslash A_{\varepsilon_{1}}\right)+F\left(g_{k_{1}},\left(\Omega \backslash A_{\varepsilon_{1}}\right) \backslash H_{k_{1}}^{\lambda}\right) \\
& +F\left(z_{k_{1}}, A_{\varepsilon_{1}} \cup\left(\Omega \backslash H_{k_{1}}^{\lambda}\right)\right) .
\end{aligned}
$$

Since

$$
\operatorname{meas}\left(\left(\Omega \backslash A_{\varepsilon_{1}}\right) \cap H_{k_{1}}^{\lambda}\right) \leq \sum_{i=1}^{N} \operatorname{meas}\left(\left(\Omega \backslash A_{\varepsilon_{1}}\right) \cap H_{i, k_{1}}^{\lambda}\right) \leq N \min \left(\varepsilon_{1}, \delta_{1}\right)
$$

from (H2), (H4) and the choice of $A_{\varepsilon_{1}}$, we get

$$
\begin{aligned}
\left|F\left(g_{k_{1}},\left(\Omega \backslash A_{\varepsilon_{1}}\right) \backslash H_{k_{1}}^{\lambda}\right)\right| & \leq \int_{\left(\Omega \backslash A_{\varepsilon_{1}}\right) \backslash H_{k_{1}}^{\lambda}}\left|A_{\alpha}^{i}\left(x, u_{0}+g_{k_{1}}, D u_{0}+D g_{k_{1}}\right) g_{k_{1}, \alpha}^{i}\right| d x \\
\leq & \int_{\left(\Omega \backslash A_{\varepsilon_{1}}\right) \backslash H_{k_{1}}^{\lambda}}\left[C_{1}\left|D u_{0}+D g_{k_{1}}\right|^{p(x)-1}\left|D g_{k_{1}}\right|\right. \\
& \left.+C_{2}\left|u_{0}+g_{k_{1}}\right|^{p(x)-1}\left|D g_{k_{1}}\right|+G(x)\left|D g_{k_{1}}\right|\right] d x
\end{aligned}
$$




$$
\begin{aligned}
& \leq \int_{\left(\Omega \backslash A_{\varepsilon_{1}}\right) \backslash H_{k_{1}}^{\lambda}}\left[C_{1}\left|D u_{0}+D g_{k_{1}}\right|^{p(x)}+C_{1}\left|D g_{k_{1}}\right|^{p(x)}+C_{2}\left|u_{0}+g_{k_{1}}\right|^{p(x)}\right. \\
& \left.+C_{2}\left|D g_{k_{1}}\right|^{p(x)}+(G(x))^{p^{\prime}(x)}+\left|D g_{k_{1}}\right|^{p(x)}\right] d x \\
& \leq \int_{\left(\Omega \backslash A_{\varepsilon_{1}}\right) \backslash H_{k_{1}}^{\lambda}}\left[C_{1} 2^{p^{*}-1}\left|D u_{0}\right|^{p(x)}+C_{1} 2^{p^{*}-1}\left|D g_{k_{1}}\right|^{p(x)}\right. \\
& +C_{1}\left|D g_{k_{1}}\right|^{p(x)}+C_{2} 2^{p^{*}-1}\left|u_{0}\right|^{p(x)}+C_{2} 2^{p^{*}-1}\left|g_{k_{1}}\right|^{p(x)} \\
& \left.+C_{2}\left|D g_{k_{1}}\right|^{p(x)}+(G(x))^{p^{\prime}(x)}+\left|D g_{k_{1}}\right|^{p(x)}\right] d x \\
& \leq 2^{p^{*}-1} \eta\left(\operatorname{meas}\left(\left(\Omega \backslash A_{\varepsilon_{1}}\right) \backslash H_{k_{1}}^{\lambda}\right)\right) \\
& +2^{p^{*}-1}\left(C_{1}+C_{2}+1\right) \int_{\left(\Omega \backslash A_{\varepsilon_{1}}\right) \backslash H_{k_{1}}^{\lambda}}\left(\left|g_{k_{1}}\right|^{p(x)}+\left|D g_{k_{1}}\right|^{p(x)}\right) d x \\
& \leq 2^{p^{*}-1} C\left(n, \Omega, C_{1}+C_{2}\right) \int_{\left(\Omega \backslash A_{\varepsilon_{1}}\right) \backslash H_{k_{1}}^{\lambda}} \lambda^{p(x)} d x+2^{p^{*}-1} \eta\left(N \varepsilon_{1}\right) \\
& \leq 2^{p^{*}-1} C\left(n, \Omega, C_{1}+C_{2}\right) \sum_{i=1}^{N} \int_{\left(\Omega \backslash A_{\varepsilon_{1}}\right) \backslash H_{i, k_{1}}^{\lambda}}\left(M^{*} z_{k_{1}}^{i}\right)^{p(x)} d x+2^{p^{*}-1} \eta\left(N \varepsilon_{1}\right) \\
& \leq 2^{p^{*}-1} \eta\left(N \varepsilon_{1}\right)+2^{p^{*}-1} C\left(n, \Omega, C_{1}+C_{2}\right) N \varepsilon_{1}=V_{1}\left(\varepsilon_{1}\right),
\end{aligned}
$$

while

$$
\begin{aligned}
& F\left(z_{k_{1}}, A_{\varepsilon_{1}} \cup\left(\Omega \backslash H_{k_{1}}^{\lambda}\right)\right) \\
& =\int_{A_{\varepsilon_{1}} \cup\left(\Omega \backslash H_{k_{1}}^{\lambda}\right)} A_{\alpha}^{i}\left(x, u_{0}+z_{k_{1}}, D u_{0}+D z_{k_{1}}\right) z_{k_{1}, \alpha}^{i} d x \\
& =\int_{A_{\varepsilon_{1}} \cup\left(\Omega \backslash H_{k_{1}}^{\lambda}\right)} A_{\alpha}^{i}\left(x, u_{0}+z_{k_{1}}, D u_{0}+D z_{k_{1}}\right)\left(u_{0, \alpha}^{i}+z_{k_{1}, \alpha}^{i}\right) d x \\
& \quad-\int_{A_{\varepsilon_{1}} \cup\left(\Omega \backslash H_{k_{1}}^{\lambda}\right)} A_{\alpha}^{i}\left(x, u_{0}+z_{k_{1}}, D u_{0}+D z_{k_{1}}\right) u_{0, \alpha}^{i} d x \\
& \geq \int_{A_{\varepsilon_{1}} \cup\left(\Omega \backslash H_{k_{1}}^{\lambda}\right)}\left[\lambda_{0}\left|D u_{0}+D z_{k_{1}}\right|^{p(x)}-C\left|u_{0}+z_{k_{1}}\right|^{p(x)}+h(x)\right] d x \\
& \quad-\int_{A_{\varepsilon_{1}} \cup\left(\Omega \backslash H_{k_{1}}^{\lambda}\right)}\left[C_{1}\left|D u_{0}+D z_{k_{1}}\right|^{p(x)-1}\left|D u_{0}\right|\right. \\
& \left.\quad+C_{2}\left|u_{0}+z_{k_{1}}\right|^{p(x)-1}\left|D u_{0}\right|+G(x)\left|D u_{0}\right|\right] d x
\end{aligned}
$$




$$
\begin{aligned}
\geq & \left(\frac{\lambda_{0}}{2^{p^{*}-1}}-\mu\right) \int_{A_{\varepsilon_{1}} \cup\left(\Omega \backslash H_{k_{1}}^{\lambda}\right.}\left|D z_{k_{1}}\right|^{p(x)} d x \\
& -C\left(\mu, \lambda_{0}, p(\cdot), C_{1}, C_{2}, C\right) \eta\left(\operatorname{meas}\left(A_{\varepsilon_{1}} \cup\left(\Omega \backslash H_{k_{1}}^{\lambda}\right)\right)\right)
\end{aligned}
$$

where $\mu>0$ is arbitrary. Taking

$$
0<\mu<\frac{\lambda_{0}}{2^{\left(p^{*}-1\right) / 2}},
$$

we have

$$
F\left(z_{k_{1}}, A_{\varepsilon_{1}} \cup\left(\Omega \backslash H_{k_{1}}^{\lambda}\right)\right) \geq \frac{\lambda_{0}}{2^{\left(p^{*}-1\right) / 2}} \int_{A_{\varepsilon_{1}} \cup\left(\Omega \backslash H_{k_{1}}^{\lambda}\right)}\left|D z_{k_{1}}\right|^{p(x)} d x-V_{2}\left(\varepsilon_{1}\right)
$$

where $V_{1}(\varepsilon), V_{2}(\varepsilon) \rightarrow 0$ as $\varepsilon \rightarrow 0^{+}$.

Set $A_{\varepsilon_{1}} \cup\left(\Omega \backslash H_{k_{1}}^{\lambda}\right)=U_{\varepsilon_{1}, k_{1}}^{1}, \alpha_{0}=\lambda_{0} / 2^{\left(p^{*}-1\right) / 2}, V_{3}(\varepsilon)=V_{1}(\varepsilon)+V_{2}(\varepsilon)$. From (3.9)-(3.11), we get

$$
F\left(z_{k_{1}}, \Omega\right) \geq F\left(g_{k_{1}}, \Omega \backslash A_{\varepsilon_{1}}\right)+\alpha_{0} \int_{U_{\varepsilon_{1}, k_{1}}^{1}}\left|D z_{k_{1}}\right|^{p(x)} d x-V_{3}\left(\varepsilon_{1}\right) .
$$

Next, set

$$
h_{k_{1}}=g_{k_{1}}-v
$$

where $v$ is defined by (3.8). Then

$$
h_{k_{1}} \rightarrow 0 \quad * \text {-weakly in } W^{1, \infty}\left(\Omega, \mathbb{R}^{N}\right) \text { as } k_{1} \rightarrow \infty
$$

and

$$
\left\|h_{k_{1}}\right\|_{L^{\infty}\left(\Omega, \mathbb{R}^{N}\right)} \leq 2 \lambda, \quad\left\|\left|D h_{k_{1}}\right|\right\|_{L^{\infty}(\Omega)} \leq 2 C(n) \lambda .
$$

Set $G=\{x \in \Omega: v(x) \neq 0\}$. According to Acerbi and Fusco [1], we have

$$
\operatorname{meas}(G) \leq(N+1) \varepsilon_{1}
$$

and

$$
\begin{aligned}
F\left(g_{k_{1}}, \Omega \backslash\right. & \left.A_{\varepsilon_{1}}\right) \\
= & F\left(h_{k_{1}},\left(\Omega \backslash A_{\varepsilon_{1}}\right) \backslash G\right)+F\left(g_{k_{1}},\left(\Omega \backslash A_{\varepsilon_{1}}\right) \cap H_{k_{1}}^{\lambda} \cap G\right) \\
& +F\left(g_{k_{1}},\left(\Omega \backslash A_{\varepsilon_{1}}\right) \cap\left(G \backslash H_{k_{1}}^{\lambda}\right)\right) \\
= & F\left(h_{k_{1}},\left(\Omega \backslash A_{\varepsilon_{1}}\right) \backslash G\right)+F\left(z_{k_{1}},\left(\Omega \backslash A_{\varepsilon_{1}}\right) \cap H_{k_{1}}^{\lambda} \cap G\right) \\
& +F\left(g_{k_{1}},\left(\Omega \backslash A_{\varepsilon_{1}}\right) \cap\left(G \backslash H_{k_{1}}^{\lambda}\right)\right) .
\end{aligned}
$$

Define

$$
\begin{aligned}
U_{\varepsilon_{1}}^{2} & =\left(\Omega \backslash A_{\varepsilon_{1}}\right) \backslash G, \\
U_{\varepsilon_{1}, k_{1}}^{3} & =\left(\Omega \backslash A_{\varepsilon_{1}}\right) \cap H_{k_{1}}^{\lambda} \cap G, \\
U_{\varepsilon_{1}, k_{1}}^{4} & =\left(\Omega \backslash A_{\varepsilon_{1}}\right) \cap\left(G \backslash H_{k_{1}}^{\lambda}\right) .
\end{aligned}
$$


Similarly to the proof of (3.12), we get

$$
F\left(z_{k_{1}}, U_{\varepsilon_{1}, k_{1}}^{3}\right) \geq \alpha_{0} \int_{U_{\varepsilon_{1}, k_{1}}^{3}}\left|D z_{k_{1}}\right|^{p(x)} d x-V_{4}\left(\varepsilon_{1}\right)
$$

On $U_{\varepsilon_{1}, k_{1}}^{4}$, we have

$$
\int_{U_{\varepsilon_{1}, k_{1}}^{4}}\left(\left|g_{k_{1}}\right|^{p(x)}+\left|D g_{k_{1}}\right|^{p(x)}\right) d x \leq N C(n, \Omega) \varepsilon_{1} .
$$

Then similarly to the proof of (3.10), we have

$$
\begin{aligned}
\left|F\left(g_{k_{1}}, U_{\varepsilon_{1}, k_{1}}^{4}\right)\right| & \leq C\left(C_{1}, C_{2}, p(\cdot)\right) N C(n, \Omega) \varepsilon_{1}+\eta\left((N+1) \varepsilon_{1}\right) \\
& =V_{3}\left(\varepsilon_{1}\right) .
\end{aligned}
$$

From (3.13)-(3.15), we get

$$
F\left(g_{k_{1}}, \Omega \backslash A_{\varepsilon_{1}}\right) \geq F\left(h_{k_{1}}, U_{\varepsilon_{1}}^{2}\right)+\alpha_{0} \int_{U_{\varepsilon_{1}, k_{1}}^{3}}\left|D z_{k_{1}}\right|^{p(x)} d x-V_{4}\left(\varepsilon_{1}\right)-V_{5}\left(\varepsilon_{1}\right)
$$

Define

$$
U_{\varepsilon_{1}, k_{1}}^{5}=U_{\varepsilon_{1}, k_{1}}^{3} \cup U_{\varepsilon_{1}, k_{1}}^{1} .
$$

From (3.12),

$$
F\left(z_{k_{1}}, \Omega\right) \geq F\left(h_{k_{1}}, U_{\varepsilon_{1}}^{2}\right)+\alpha_{0} \int_{U_{\varepsilon_{1}, k_{1}}^{5}}\left|D z_{k_{1}}\right|^{p(x)} d x-V_{6}\left(\varepsilon_{1}\right)
$$

where $V_{6}(\varepsilon)=V_{3}(\varepsilon)+V_{4}(\varepsilon)+V_{5}(\varepsilon)$.

Choose an open set $\Omega^{\prime} \subset \Omega$ which contains $U_{\varepsilon_{1}}^{2}$ such that

$$
\left|F\left(h_{k_{1}}, \Omega^{\prime}\right)-F\left(h_{k_{1}}, U_{\varepsilon_{1}}^{2}\right)\right|<\varepsilon_{1} .
$$

In view of (3.16), we get

$$
F\left(z_{k_{1}}, \Omega\right) \geq F\left(h_{k_{1}}, \Omega^{\prime}\right)+\alpha_{0} \int_{U_{\varepsilon_{1}, k_{1}}^{5}}\left|D z_{k_{1}}\right|^{p(x)} d x-V_{7}\left(\varepsilon_{1}\right)
$$

where $V_{7}(\varepsilon)=V_{6}(\varepsilon)+\varepsilon$.

Next approximate $\Omega^{\prime}$ by hypercubes with edges parallel to the coordinate axes, i.e. construct

$$
\left\{\begin{array}{l}
H_{j}=\bigcup_{s=1}^{I_{j}} D_{j, s} \\
\operatorname{meas}\left(\Omega^{\prime} \backslash H_{j}\right) \rightarrow 0 \quad \text { as } j \rightarrow \infty \\
\operatorname{meas}\left(D_{j, s}\right)=1 / 2^{n j}, \quad 1 \leq s \leq I_{j} \\
H_{j} \subset \Omega^{\prime}
\end{array}\right.
$$


Let $j>0$ be so large that for all $k_{1}>0$,

$$
\left|F\left(h_{k_{1}}, \Omega^{\prime}\right)-F\left(h_{k_{1}}, H_{j}\right)\right|<\varepsilon_{1}, \quad \int_{\Omega^{\prime} \backslash H_{j}}\left|D h_{k_{1}}\right|^{p(x)} d x<\varepsilon_{1}
$$

and

$$
\operatorname{meas}\left(\Omega^{\prime} \backslash H_{j}\right)<\min \left(\varepsilon_{1}, \delta_{1}\right)
$$

Then

$$
F\left(z_{k_{1}}, \Omega\right) \geq F\left(h_{k_{1}}, H_{j}\right)+\alpha_{0} \int_{\substack{U_{\varepsilon_{1}, k_{1}}^{5} \\ f_{1}}}\left|D z_{k_{1}}\right|^{p(x)} d x-V_{8}\left(\varepsilon_{1}\right)
$$

where $V_{8}(\varepsilon)=V_{7}(\varepsilon)+\varepsilon$.

Let

$$
M=2 C(n) \lambda \geq\left\|\left|D h_{k_{1}}\right|\right\|_{L^{\infty}(\Omega)}
$$

and $\alpha>0$ be so large that for $E=\left\{x \in \Omega^{\prime}: a(x) \leq \alpha\right\}$, we have

$$
\operatorname{meas}\left(\Omega^{\prime} \backslash E\right) \leq \varepsilon_{1} / M, \quad \int_{\Omega^{\prime} \backslash E} a(x) d x \leq \varepsilon_{1}
$$

where

$$
a(x)=2^{p^{*}-1}\left[\left(1+C_{1}+C_{2}\right)\left|D u_{0}(x)\right|^{p(x)}+C_{2}\left|u_{0}(x)\right|^{p(x)}+(G(x))^{p^{\prime}(x)}\right] .
$$

For $x \in \Omega, s \in \mathbb{R}^{N}, \xi \in M^{N \times n}$, define

$$
f(x, s, \xi):=A_{\alpha}^{i}\left(x, u_{0}(x)+s, D u_{0}(x)+\xi\right) \xi_{\alpha}^{i} .
$$

By Lemma 2.1, there exists a compact subset $K \subset H_{j}$ such that $f(x, s, \xi)$ is continuous on $K \times \mathbb{R}^{N} \times M^{N \times n}$ and

$$
\operatorname{meas}\left(H_{j} \backslash K\right)<\frac{\varepsilon_{1}}{\alpha+M} .
$$

Divide each $D_{j, s}$ into $2^{n m}$ hypercubes $Q_{h, s, j}^{m}$ with edge length $2^{-j m}, 1 \leq$ $h \leq 2^{n m}$. For all $j, s, m, h$, take $x_{h, s, j}^{m} \in Q_{h, s, j}^{m} \cap K \cap E$ (if this set is empty, take $x_{h, s, j}^{m} \in Q_{h, s, j}^{m}$ ) such that

$$
a\left(x_{h, s, j}^{m}\right) \operatorname{meas}\left(Q_{h, s, j}^{m}\right) \leq \int_{Q_{h, s, j}^{m}} a(x) d x .
$$

Then

(3.19) $\quad F\left(h_{k_{1}}, H_{j}\right)$

$$
=F\left(h_{k_{1}}, H_{j} \cap K \cap E\right)+F\left(h_{k_{1}}, H_{j} \backslash E\right)+F\left(h_{k_{1}},\left(H_{j} \cap E\right) \backslash K\right)
$$




$$
\begin{aligned}
\geq & F\left(h_{k_{1}}, H_{j} \cap K \cap E\right)-\int_{H_{j} \backslash E} a(x) d x-\int_{\left(H_{j} \cap E\right) \backslash K} a(x) d x \\
& -2^{p^{*}-1}\left(1+C_{1}+C_{2}\right)\left(\int_{H_{j} \backslash E}\left[\left|D h_{k_{1}}\right|^{p(x)}+\left|h_{k_{1}}\right|^{p(x)}\right] d x\right. \\
& \left.+\int_{\left(H_{j} \cap E\right) \backslash K}\left[\left|D h_{k_{1}}\right|^{p(x)}+\left|h_{k_{1}}\right|^{p(x)}\right] d x\right) \\
= & F\left(h_{k_{1}}, H_{j} \cap K \cap E\right)-V_{9}\left(\varepsilon_{1}\right) \\
= & a_{k_{1}}^{j}+b_{k_{1}}^{m, j}+c_{k_{1}}^{m, j}+d_{k_{1}}^{m, j}-V_{9}\left(\varepsilon_{1}\right)
\end{aligned}
$$

where

$$
\begin{aligned}
a_{k_{1}}^{j} & =\int_{H_{j} \cap K \cap E}\left[f\left(x, h_{k_{1}}(x), D h_{k_{1}}(x)\right)-f\left(x, 0, D h_{k_{1}}(x)\right)\right] d x \\
b_{k_{1}}^{m, j} & =\sum_{h, s} \int_{Q_{h, s, j}^{m} \cap K \cap E}\left[f\left(x, 0, D h_{k_{1}}(x)\right)-f\left(x_{h, s, j}^{m}, 0, D h_{k_{1}}(x)\right)\right] d x \\
c_{k_{1}}^{m, j} & =\sum_{h, s} \int_{Q_{h, s, j}^{m}} f\left(x_{h, s, j}^{m}, 0, D h_{k_{1}}(x)\right) d x \\
d_{k_{1}}^{m, j} & =-\sum_{h, s} \int_{Q_{h, s, j}^{m} \backslash(K \cap E)} f\left(x_{h, s, j}^{m}, 0, D h_{k_{1}}(x)\right) d x .
\end{aligned}
$$

By the uniform continuity of $f$ on bounded sets of $K \times \mathbb{R}^{N} \times M^{N \times n}$ and (3.7), we have

$$
\lim _{k_{1} \rightarrow \infty} a_{k_{1}}^{j}=0, \quad \lim _{k_{1} \rightarrow \infty} F\left(z_{k_{1}}, \Omega\right)=0
$$

and the pointwise convergence of $u_{0}\left(x_{h, s, j}^{m}\right), D u_{0}\left(x_{h, s, j}^{m}\right)$ implies

$$
\lim _{m \rightarrow \infty} h_{k_{1}}^{m, j}=0
$$

uniformly with respect to $k_{1}$, for fixed $j$, and

$$
\begin{aligned}
\left|d_{k_{1}}^{m, j}\right| & \leq \sum_{h, s} \int_{Q_{h, s, j}^{m} \backslash(K \cap E)}\left[a\left(x_{h, s, j}^{m}\right)+2^{p^{*}}\left(1+C_{1}+C_{2}\right) M\right] d x \\
& \leq C(\alpha+M) \operatorname{meas}\left(\left(H_{j} \cap E\right) \backslash K\right)+C \int_{H_{j} \backslash E}[a(x)+M] d x \\
& \leq C\left(C_{1}, C_{2}, p(\cdot)\right) \varepsilon_{1} .
\end{aligned}
$$

Now we suppose that $m$ is so large that $\left|b_{k_{1}}^{m, j}\right|<\varepsilon_{1}$ for each $k_{1}>0$ and there exists $\bar{k}_{1}>0$ such that $F\left(z_{k_{1}}, \Omega\right)<\varepsilon_{1},\left|a_{k_{1}}^{j}\right|<\varepsilon_{1}$ for $k_{1}>\bar{k}_{1}$. Therefore from $(3.7),(3.18)$ and $(3.19)$, we have 


$$
\begin{aligned}
\varepsilon_{1} \geq & F\left(z_{k_{1}}, \Omega\right) \\
\geq & c_{k_{1}}^{m, j}+\alpha_{0} \int_{U_{\varepsilon_{1}, k_{1}}^{5}}\left|D z_{k_{1}}\right|^{p(x)} d x-V_{8}\left(\varepsilon_{1}\right)-V_{9}\left(\varepsilon_{1}\right) \\
& -2 \varepsilon_{1}-C\left(C_{1}, C_{2}, p(\cdot)\right) \varepsilon_{1} \\
= & c_{k_{1}}^{m, j}+\alpha_{0} \int_{U_{\varepsilon_{1}, k_{1}}^{5}}\left|D z_{k_{1}}\right|^{p(x)} d x-V_{10}\left(\varepsilon_{1}\right) .
\end{aligned}
$$

As $h_{k_{1}} \rightarrow 0$ weakly in $W^{1, \infty}\left(\Omega, \mathbb{R}^{N}\right)$ as $k_{1} \rightarrow \infty$, we obtain

$$
R_{h, s, j}^{k_{1}, m}=\left\|\left|h_{k_{1}}\right|\right\|_{L^{\infty}\left(Q_{h, s, j}^{m}\right)} \rightarrow 0 \quad \text { as } k_{1} \rightarrow \infty
$$

for fixed $m$.

Define a hypercube $E_{h, s, j}^{k_{1}, m}$ contained in $Q_{h, s, j}^{m}$ with edge length $1 / 2^{j m}-$ $2 R_{h, s, j}^{k_{1}, m}$ such that

$$
\operatorname{dist}\left(\partial Q_{h, s, j}^{m}, E_{h, s, j}^{k_{1}, m}\right)=R_{h, s, j}^{k_{1}, m}
$$

Next define

$$
f_{k_{1}}(x)= \begin{cases}0, & x \in \partial Q_{h, s, j}^{m}, \\ h_{k_{1}}(x), & x \in E_{h, s, j}^{k_{1}, m}\end{cases}
$$

Since $f_{k_{1}}$ is a Lipschitz mapping on the set where it is defined and its Lipschitz constant is no more than $2 C(n) \lambda$, by Lemma $2.7, f_{k_{1}}$ can be extended to the whole $Q_{h, s, j}^{m}$, where it is also a Lipschitz mapping with the same Lipschitz constant. We still denote the extension by $f_{k_{1}}$ and suppose that it is defined on the whole $H_{j}$. Then by [4],

$$
D f_{k_{1}}(x)-D h_{k_{1}}(x) \rightarrow 0 \quad \text { a.e. on } H_{j} \text {. }
$$

So there exists a $\overline{\bar{k}}_{1}>\bar{k}_{1}$ such that for all $k_{1}>\overline{\bar{k}}_{1}$, we have

$$
\begin{gathered}
\int_{H_{j}}\left|D f_{k_{1}}-D h_{k_{1}}\right|^{p(x)} d x \leq \frac{\varepsilon_{1}}{2}, \\
\left|\sum_{h, s} \int_{Q_{h, s, j}^{m}}\left[f\left(x_{h, s, j}^{m}, 0, D h_{k_{1}}\right)-f\left(x_{h, s, j}^{m}, 0, D f_{k_{1}}\right)\right] d x\right| \leq \frac{\varepsilon_{1}}{2} .
\end{gathered}
$$

In view of $(\mathrm{H} 5)$,

$$
\begin{aligned}
c_{k_{1}}^{m, j} & =\sum_{h, s} \int_{Q_{h, s, j}^{m}} f\left(x_{h, s, j}^{m}, 0, D h_{k_{1}}\right) d x \geq \sum_{h, s} \int_{Q_{h, s, j}^{m}} f\left(x_{h, s, j}^{m}, 0, D f_{k_{1}}\right) d x-\frac{\varepsilon_{1}}{2} \\
& \geq \sum_{h, s} \nu \int_{Q_{h, s, j}^{m}}\left|D f_{k_{1}}\right|^{p(x)} d x-\frac{\varepsilon_{1}}{2} \geq \frac{\nu}{2^{p^{*}-1}} \int_{H_{j}}\left|D h_{k_{1}}\right|^{p(x)} d x-\frac{(\nu+1) \varepsilon_{1}}{2} .
\end{aligned}
$$


Thus in (3.20) for $k_{1} \geq \overline{\bar{k}}_{1}$, we have

$$
\varepsilon_{1} \geq \alpha_{0} \int_{U_{\varepsilon_{1}, k_{1}}^{5}}\left|D z_{k_{1}}\right|^{p(x)} d x+\frac{\nu}{2^{p^{*}-1}} \int_{H_{j}}\left|D h_{k_{1}}\right|^{p(x)} d x-\frac{(\nu+1) \varepsilon_{1}}{2}-V_{10}\left(\varepsilon_{1}\right) .
$$

Set

$$
K(\varepsilon)=\frac{V_{10}(\varepsilon)+(\nu+3) \varepsilon / 2}{\min \left(\alpha_{0}, \nu / 2^{p^{*}-1}\right)}
$$

Then

$$
\int_{H_{j}}\left|D h_{k_{1}}\right|^{p(x)} d x+\int_{U_{\varepsilon_{1}, k_{1}}^{5}}\left|D z_{k_{1}}\right|^{p(x)} d x \leq K\left(\varepsilon_{1}\right)
$$

for $k_{1}>\overline{\bar{k}}_{1}$. From (3.17) and (3.21), we deduce that

$$
\int_{\Omega^{\prime}}\left|D h_{k_{1}}\right|^{p(x)} d x \leq K\left(\varepsilon_{1}\right)+\varepsilon_{1}, \quad \int_{U_{\varepsilon_{1}, k_{1}}^{5}}\left|D z_{k_{1}}\right|^{p(x)} d x \leq K\left(\varepsilon_{1}\right) .
$$

According to the definition of $\Omega^{\prime}$, we have

$$
\int_{U_{\varepsilon_{1}}^{2}}\left|D g_{k_{1}}\right|^{p(x)} d x \leq K\left(\varepsilon_{1}\right)+\varepsilon_{1}
$$

Since $D g_{k_{1}}(x)=D z_{k_{1}}(x)$ for each $x \in H_{k_{1}}^{\lambda}$, we get

$$
\int_{U_{\varepsilon_{1}}^{2} \cap H_{k_{1}}^{\lambda}}\left|D z_{k_{1}}\right|^{p(x)} d x \leq K\left(\varepsilon_{1}\right)+\varepsilon_{1} .
$$

By the definition of $U_{\varepsilon_{1}}^{2}$ and $U_{\varepsilon_{1}, k_{1}}^{5}$, it is immediate that

$$
\left(U_{\varepsilon_{1}}^{2} \cap H_{k_{1}}\right) \cup U_{\varepsilon_{1}, k_{1}}^{5}=\Omega
$$

which implies that

$$
\int_{\Omega}\left|D z_{k_{1}}\right|^{p(x)} d x \leq 2 K\left(\varepsilon_{1}\right)+\varepsilon_{1}=W\left(\varepsilon_{1}\right)
$$

where $W(\varepsilon) \rightarrow 0$ as $\varepsilon \rightarrow 0^{+}$.

For $\varepsilon_{2}>0$ and the sequence $\left\{z_{k_{1}}\right\}$, repeating the above arguments we can extract a subsequence of $\left\{z_{k_{1}}\right\}$, denoted by $\left\{z_{k_{2}}\right\}$, such that

$$
\int_{\Omega}\left|D z_{k_{2}}\right|^{p(x)} d x \leq W\left(\varepsilon_{2}\right)
$$

whenever $k_{2}>\overline{\bar{k}}_{2}$ for some $\overline{\bar{k}}_{2}$. If $\left\{z_{k_{n}}\right\}$ has been obtained, repeating the above process, we can extract a subsequence of $\left\{z_{k_{n}}\right\}$, denoted by $\left\{z_{k_{n+1}}\right\}$, which satisfies

$$
\int_{\Omega}\left|D z_{k_{n+1}}\right|^{p(x)} d x \leq W\left(\varepsilon_{n+1}\right)
$$


whenever $k_{n+1}>\overline{\bar{k}}_{n+1}$ for some $\overline{\bar{k}}_{n+1}$. Finally, by a diagonal argument we get a subsequence $\left\{z_{k_{i}}\right\}_{i=1}^{\infty}$ of $\left\{z_{k}\right\}$ which satisfies

$$
\int_{\Omega}\left|D z_{k_{i}}\right|^{p(x)} d x \rightarrow 0 \quad \text { as } i \rightarrow \infty .
$$

By Lemma 2.9, we have

$$
\left\|\left|D z_{k_{i}}\right|\right\|_{L^{p(\cdot)}(\Omega)} \rightarrow 0 \quad \text { as } i \rightarrow \infty
$$

and furthermore $\left\{z_{k_{i}}\right\}_{i=1}^{\infty}$ converges to zero strongly in $W_{0}^{1, p(\cdot)}\left(\Omega, \mathbb{R}^{N}\right)$ as $i \rightarrow \infty$. This completes the proof of Theorem 3.1.

If we choose $p(x) \equiv p, 1<p<\infty$, then we get

Corollary 3.1. Assume the following conditions:

(G1) The same as (H1).

(G2) $|A(x, s, \xi)| \leq C_{1}|\xi|^{p-1}+C_{2}|s|^{p-1}+G(x)$, where $G \in L^{p^{\prime}}(\Omega), C_{1}, C_{2}$ $\geq 0$ and $C_{2}$ is small, $1 / p+1 / p^{\prime}=1$.

(G3) $\quad|B(x, s, \xi)| \leq C_{1}^{\prime}|\xi|^{p-1}+C_{2}^{\prime}|s|^{p-1}+\bar{G}(x)$, where $\bar{G} \in L^{p^{\prime}}(\Omega), C_{1}^{\prime}, C_{2}^{\prime}$ $\geq 0$ and are small.

(G4) $\quad A_{\alpha}^{i}(x, s, \xi) \xi_{\alpha}^{i} \geq \lambda_{0}|\xi|^{p}-C|s|^{p}+h(x)$, where $\lambda_{0}>0, C \geq 0$ is small and $h \in L^{1}(\Omega)$.

(G5) For almost every $x_{0} \in \Omega, s_{0} \in \mathbb{R}^{N}$, the mapping $\xi \mapsto A\left(x_{0}, s_{0}, \xi\right)$ satisfies

$$
\int_{G} A_{\alpha}^{i}\left(x_{0}, s_{0}, \xi_{0}+D z(x)\right) z_{, \alpha}^{i}(x) d x \geq \nu \int_{G}|D z(x)|^{p} d x
$$

for each $\xi_{0} \in M^{N \times n}, G \subset \mathbb{R}^{n}, z \in C_{0}^{1}\left(G, \mathbb{R}^{N}\right)$ where $\nu>0$.

Then the system (1.1)-(1.2) has at least one weak solution which satisfies

$$
u \in W_{0}^{1, p}\left(\Omega, \mathbb{R}^{N}\right)
$$

and

$$
\int_{\Omega}\left[A_{\alpha}^{i}(x, u, D u) z_{, \alpha}^{i}(x)+B^{i}(x, u, D u) z^{i}(x)\right] d x=0
$$

for all $z \in W_{0}^{1, p}\left(\Omega, \mathbb{R}^{N}\right)$.

Acknowledgements. The author thanks the referees for careful reading and helpful suggestions on the improvement of the manuscript.

\section{References}

[1] E. Acerbi and N. Fusco, Semicontinuity problems in the calculus of variations, Arch. Rational Mech. Anal. 86 (1984), 125-135. 
[2] E. Acerbi and N. Fusco, Partial regularity under anisotropic $(p, q)$ growth conditions, J. Differential Equations 107 (1994), 46-67.

[3] - - - A transmission problem in the calculus of variations, Calc. Var. 2 (1994), $1-16$.

[4] B. Dacorogna, Weak Continuity and Weak Lower Semicontinuity of Nonlinear Functionals, Lecture Notes in Math. 922, Springer, 1982.

[5] G. Eisen, A selection lemma for sequences of measurable sets and lower semicontinuity of multiple integrals, Manuscripta Math. 27 (1979), 73-79.

[6] I. Ekeland and R. Temam, Convex Analysis and Variational Problems, NorthHolland, Amsterdam, 1976.

[7] X. Fan and Z. Dun, A class of De Giorgi type and Hölder continuity of minimizers of variational functionals with $m(x)$-growth conditions, Nonlinear Anal. 36 (1999), 295-318.

[8] - - - The quasi-minimizer of integral functionals with $m(x)$ growth conditions, ibid. 39 (2000), 807-816.

[9] N. Fusco and C. Sbordone, Some remarks on the regularity of minima of anisotropic integrals, Comm. Partial Differential Equations 18 (1993), 153-167.

[10] M. Giaquinta, Growth conditions and regularity, a counterexample, Manuscripta Math. 59 (1987), 245-248.

[11] O. Kováčik and J. Rákosnik, On spaces $L^{p(x)}$ and $W^{k, p(x)}$, Czechoslovak Math. J. 41 (1991), 592-618.

[12] F. C. Liu, A Luzin type property of Sobolev functions, Indiana Univ. Math. J. 26 (1977), 645-651.

[13] P. Marcellini, Regularity of minimizers of integrals of the calculus of variations with nonstandard growth conditions, Arch. Rational Mech. Anal. 105 (1989), 267-284.

[14] - Regularity and existence of solutions of elliptic equations with p, q-growth conditions, J. Differential Equations 90 (1991), 1-30.

[15] C. B. Morrey Jr., Multiple Integrals in the Calculus of Variations, Springer, New York, 1966.

[16] V. V. Zhikov and X. Fan, Lavrentiev phenomenon and estimation of Mayer type for some nonlinear equations of non-standard type, preprint.

Department of Mathematics

Harbin Institute of Technology

Harbin 150001, P.R. China

E-mail: fuyq@371.net

Received December 4, 2000

Revised version January 21, 2002 